\title{
HSP27 Alleviates Cardiac Aging in Mice via a Mechanism Involving Antioxidation and Mitophagy Activation
}

\author{
Shenglan Lin, ${ }^{1}$ Yana Wang, ${ }^{1}$ Xiaojin Zhang, ${ }^{1}$ Qiuyue Kong, ${ }^{2}$ Chuanfu Li, ${ }^{3}$ \\ Yuehua Li, ${ }^{4}$ Zhengnian Ding, ${ }^{2}$ and Li Liu ${ }^{1}$ \\ ${ }^{1}$ Department of Geriatrics, First Affiliated Hospital with Nanjing Medical University, Nanjing 210029, China \\ ${ }^{2}$ Department of Anesthesiology, First Affiliated Hospital with Nanjing Medical University, Nanjing 210029, China \\ ${ }^{3}$ Department of Surgery, East Tennessee State University, Johnson City, TN 37614, USA \\ ${ }^{4}$ Department of Pathophysiology, Nanjing Medical University, Nanjing 210029, China \\ Correspondence should be addressed to Li Liu; liuli@njmu.edu.cn
}

Received 30 September 2015; Revised 30 January 2016; Accepted 22 February 2016

Academic Editor: Alessandra Ricelli

Copyright (c) 2016 Shenglan Lin et al. This is an open access article distributed under the Creative Commons Attribution License, which permits unrestricted use, distribution, and reproduction in any medium, provided the original work is properly cited.

\begin{abstract}
Aging-induced cardiac dysfunction is a prominent feature of cardiac aging. Heat shock protein 27 (HSP27) protects cardiac function against ischemia or chemical challenge. We hypothesized that HSP27 attenuates cardiac aging. Transgenic (Tg) mice with cardiacspecific expression of the HSP27 gene and wild-type (WT) littermates were employed in the experiments. Echocardiography revealed a significant decline in the cardiac function of old WT mice compared with young WT mice. In striking contrast, the aging-induced impairment of cardiac function was attenuated in old Tg mice compared with old WT mice. Levels of cardiac aging markers were lower in old Tg mouse hearts than in old WT mouse hearts. Less interstitial fibrosis and lower contents of reactive oxygen species and ubiquitin-conjugated proteins were detected in old Tg hearts than in old WT hearts. Furthermore, old Tg hearts demonstrated lower accumulation of LC3-II and p62 than old WT hearts. Levels of Atg13, Vps34, and Rab7 were also higher in old Tg hearts than in old WT hearts. Additionally, old Tg hearts had higher levels of PINK1 and Parkin than old WT hearts, suggesting that mitophagy was activated in old Tg hearts. Taken together, HSP27 alleviated cardiac aging and this action involved antioxidation and mitophagy activation.
\end{abstract}

\section{Introduction}

Intrinsic cardiac aging is defined as slowly progressive functional declines and structural changes with age, in the absence of major cardiovascular risks such as hypertension, diabetes, hypercholesterolemia, and smoking [1, 2]. However, intrinsic cardiac aging can increase the vulnerability of the heart to both endogenous and exogenous stressors, ultimately increasing cardiovascular mortality and morbidity in elderly individuals $[1,2]$. Therefore, interventions to combat cardiac aging not only will improve the healthspan of the elderly, but also can extend their lifespan by delaying cardiovascular disease-related deaths [3].

The phenotypes of the aging heart have been well characterized; however, the molecular mechanisms underlying cardiac aging are not fully understood [3]. Studies indicate that the pathogenesis of cardiac aging involves multiple molecular mechanisms, including oxidative stress, impaired autophagy, metabolic changes, dysregulated calcium homeostasis, and activation of neurohormonal signaling $[3,4]$. Indeed, the reactive oxygen species (ROS) content significantly increases in the aged heart, while mitochondrial overexpression of catalase (an important antioxidative enzyme) improves the aging-induced decline in cardiac function and prolongs the lifespan of mice [5, 6]. Intracellular ROS in the aged heart are mainly generated from damaged mitochondria [1]. In normal conditions, damaged mitochondria are selectively degraded through autophagy, or mitophagy, a term coined by Lemasters $[1,7]$. Unfortunately, autophagy is progressively impaired over time [8].

Heat shock protein 27 (HSP27) is an ubiquitously expressed member of the small heat shock protein subfamily 
with a molecular weight of $27 \mathrm{kDa}$ in humans and $25 \mathrm{kDa}$ in rodents. Studies demonstrated the involvement of HSP27 in various biological functions, including the responses to oxidative stress, heat shock, and hypoxic/ischemia injury [9, 10]. Of particular interest to this study, we and others showed that overexpression of HSP27 protects cardiac function against cardiac injuries induced by ischemia/reperfusion, myocardial infarction, inflammation, and doxorubicin [9, 11-13]. The mechanisms that contribute to cardioprotection by HSP27 involve the antioxidative capacity, suppression of inflammatory responses, improvement of cardiomyocyte survival, and activation of autophagy and mitochondrial activity $[9,11-17]$. It is possible, therefore, that overexpression of HSP27 protects the heart from aging-induced injury.

In this study, we examined the effects of HSP27 on cardiac aging using transgenic (Tg) mice with cardiacspecific expression of HSP27. We observed an improvement in cardiac function and decreases in the levels of cardiac aging markers in old Tg mice compared with age-matched wildtype (WT) controls. This action of HSP27 involves the antioxidative capacity and activation of mitochondrial autophagy (mitophagy). Our results suggest that management of HSP27 expression may serve as an alternative intervention to alleviate cardiac aging.

\section{Materials and Methods}

2.1. Antibodies and Reagents. A primary antibody against HSP27 was obtained from Stressgen (Victoria, British Columbia, Canada). Antibodies against p16, p53, Atg13, Nix, phosphor-p53 (Ser15), p21, and GAPDH were obtained from Bioworld Technology Inc. (Louis Park, MN). Antibodies against LC3, p62, Rab7, and Vps34 were from Cell Signaling Technology (Beverly, MA). Antibodies against Parkin, PINK1, and BNIP3 were from Abcam (Cambridge, MA). An antibody against ubiquitin was from Santa Cruz Biotechnology (Dallas, TX). An antibody against dinitrophenyl was from Sigma (Saint Louis, MO). A BCA protein assay kit was obtained from Pierce (Rockford, IL). $2^{\prime}, 7^{\prime}$-Dichlorofluorescein (DCFH) was obtained from Merck (Darmstadt, Germany).

2.2. Animals. Tg mice with cardiac-specific expression of the human HSP27 gene (HSP27 Tg) driven by the $\alpha$-MHC promoter were developed using mice with a CBA/BL6 genetic background and back-crossed with C57BL/6 mice for more than ten generations as described previously $[9,13]$. In the experiments, 24-month-old HSP27 Tg mice and gendermatched WT littermates served as old mice. In echocardiographic measurements, 2-month-old HSP27 Tg mice and gender-matched WT littermates served as young controls. Mice were bred and maintained at the Model Animal Research Center of Nanjing University and maintained in the Animal Laboratory Resource Facility at Nanjing University. All the experiments were performed in compliance with the Guide for the Care and Use of Laboratory Animals published by the US National Institutes of Health (NIH Publication, 8th Edition, 2011). The animal care and experimental protocols were approved by the Nanjing University Committee on
Animal Care. All experiments were performed in compliance with the international guidelines on the ethical use of animals.

2.3. Echocardiography. Two-dimensional echocardiographic measurements were performed using the Vevo 770 system equipped with a $35 \mathrm{MHz}$ transducer (Visualsonics, Toronto, Canada) as in our previous methods [18]. Mice were anaesthetized by inhalation of $1.5-2 \%$ isoflurane. The measurements were performed by an observer blinded to the treatment. The parameters were obtained in M-mode tracings at the papillary muscle level or pulse doppler evaluation of the bicuspid valve and averaged using at least five continuous cardiac cycles.

2.4. Masson's Trichrome Staining. Heart tissues at the papillary muscle level were collected for paraffin sectioning. Masson trichrome staining was used to analyze fibrosis according to previously described methods [18]. The staining was observed using a microscope at a magnification of 400x. The percentage areas of fibrosis in the ventricular myocardium were measured using a software program (Olympus, Japan).

2.5. DCFH Assay. ROS levels were measured using the fluorescent indicator DCFH, as previously described $[9,19]$. When DCFH is added to a tissue homogenate, ROS in the homogenate will lead to the oxidation of DCFH, producing the fluorescent product DCF. In our experiments, $1 \mu \mathrm{M}$ DCFH was incubated in a volume of $1 \mathrm{~mL}$ containing $10 \mu \mathrm{g}$ of cardiac cytosolic proteins, and fluorescence was recorded following incubation for $1 \mathrm{~h}$ using a fluorometer (Synergy $\mathrm{HT}, \mathrm{BIO}-\mathrm{TEK})$ at an excitation wavelength of $485 \mathrm{~nm}$ and an emission wavelength of $535 \mathrm{~nm}$.

2.6. Protein Carbonylation. Ventricular tissues were homogenized in lysis buffer $(0.3 \mathrm{M}$ sucrose, $0.03 \mathrm{M}$ nicotinamide, and $0.02 \mathrm{M}$ EDTA, $\mathrm{pH} 7.4$ ) and centrifuged at $10,000 \times \mathrm{g}$ for $5 \mathrm{~min}$ at $4^{\circ} \mathrm{C}$. A volume of $500 \mu \mathrm{L}$ containing $100 \mu \mathrm{g}$ of cytosolic proteins was incubated with $2 \mathrm{mM}$ DNPH in the dark for $1 \mathrm{~h}$. The $\mathrm{DNPH}$-treated proteins were used to detect protein carbonylation by Western blotting.

2.7. Immunofluorescence Staining. Hearts were harvested, fixed in $4 \%$ paraformaldehyde overnight, and processed for paraffin-embedded sectioning. The sections were then dewaxed, rehydrated, subjected to antigen retrieval, and blocked. Subsequently, the sections were incubated with the appropriate primary antibodies at $4^{\circ} \mathrm{C}$ overnight, followed by FITC- or Cy3-conjugated secondary antibodies for $60 \mathrm{~min}$ at room temperature. $\alpha$-actinin was used to indicate cardiomyocytes. Hoechst 33342 was used to counterstain nuclei. The staining was observed and images were captured using a fluorescence microscope at a magnification of 400x. In LC3-II staining experiments, LC3-II granules were counted and expressed as the number of LC3-II dots per $\mathrm{mm}^{2}$ of myocardial tissues.

2.8. Western Blotting. Hearts were collected from 24-monthold mice. Western blotting of these samples was performed 


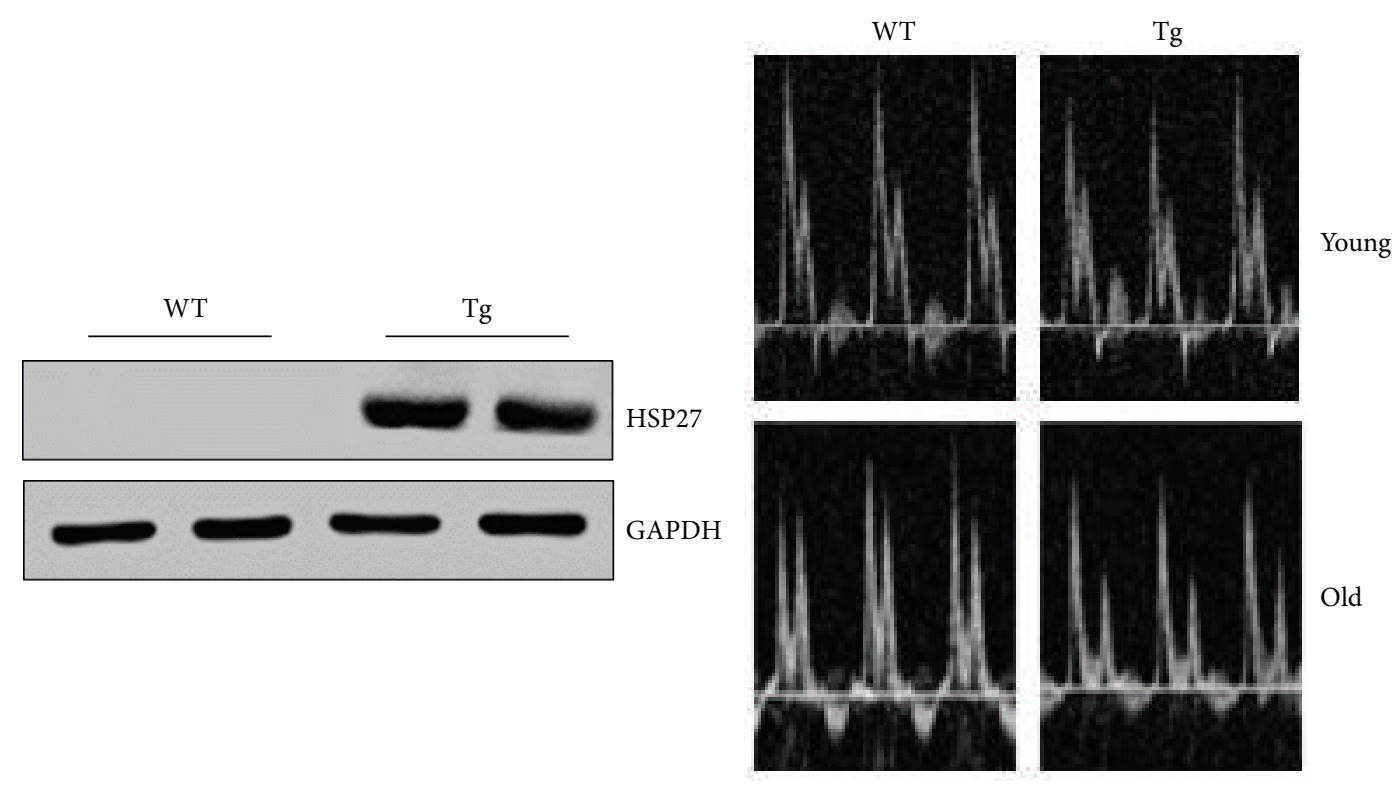

(a)

(b)
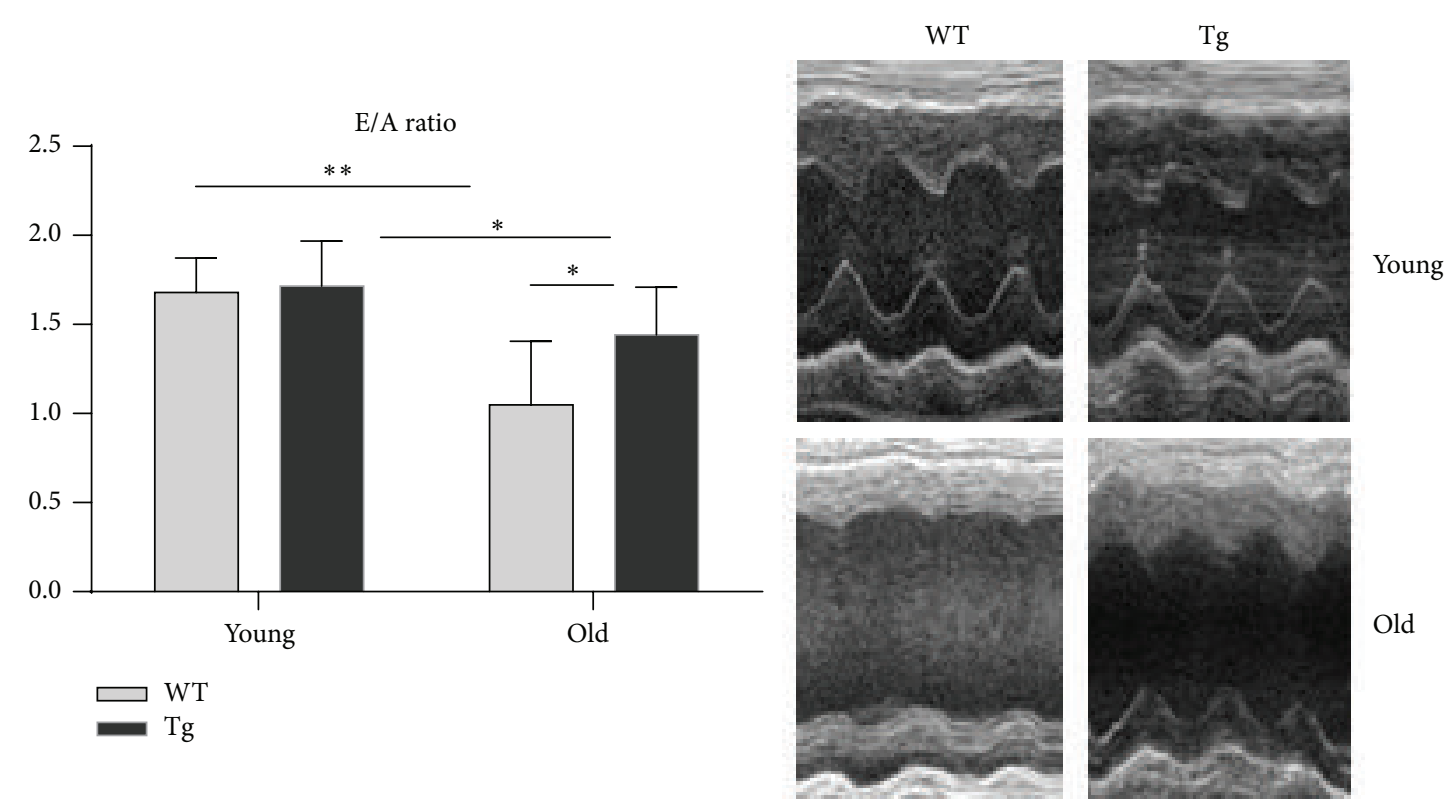

(c)

(d)
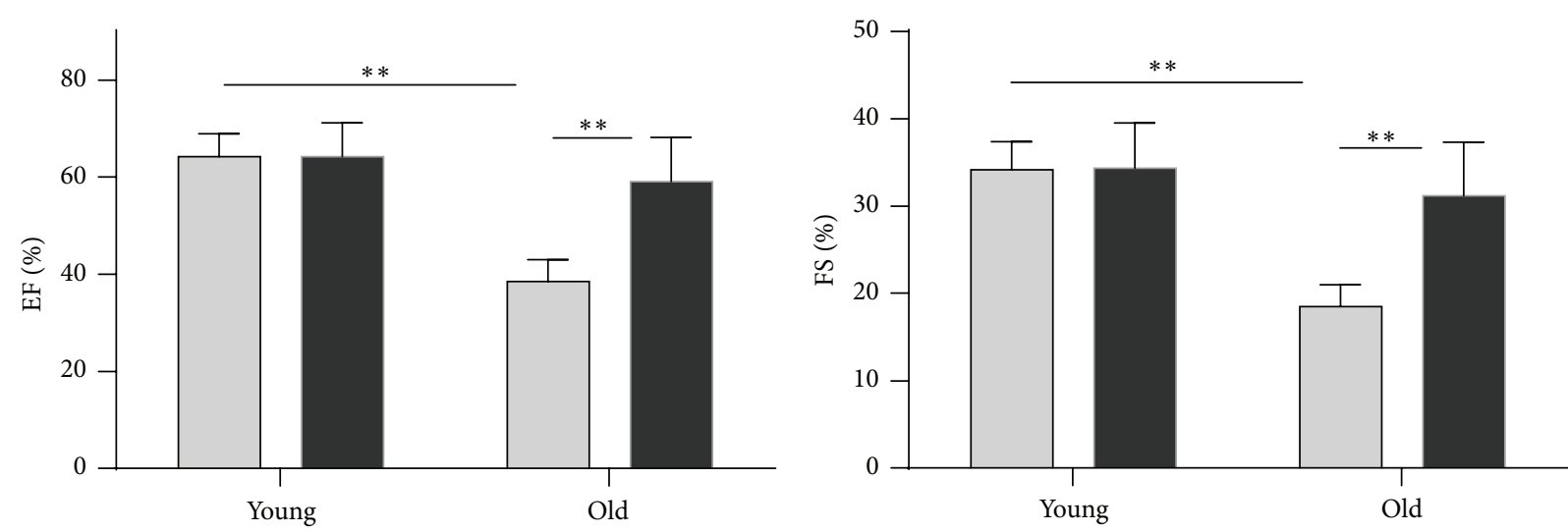

Figure 1: Continued. 

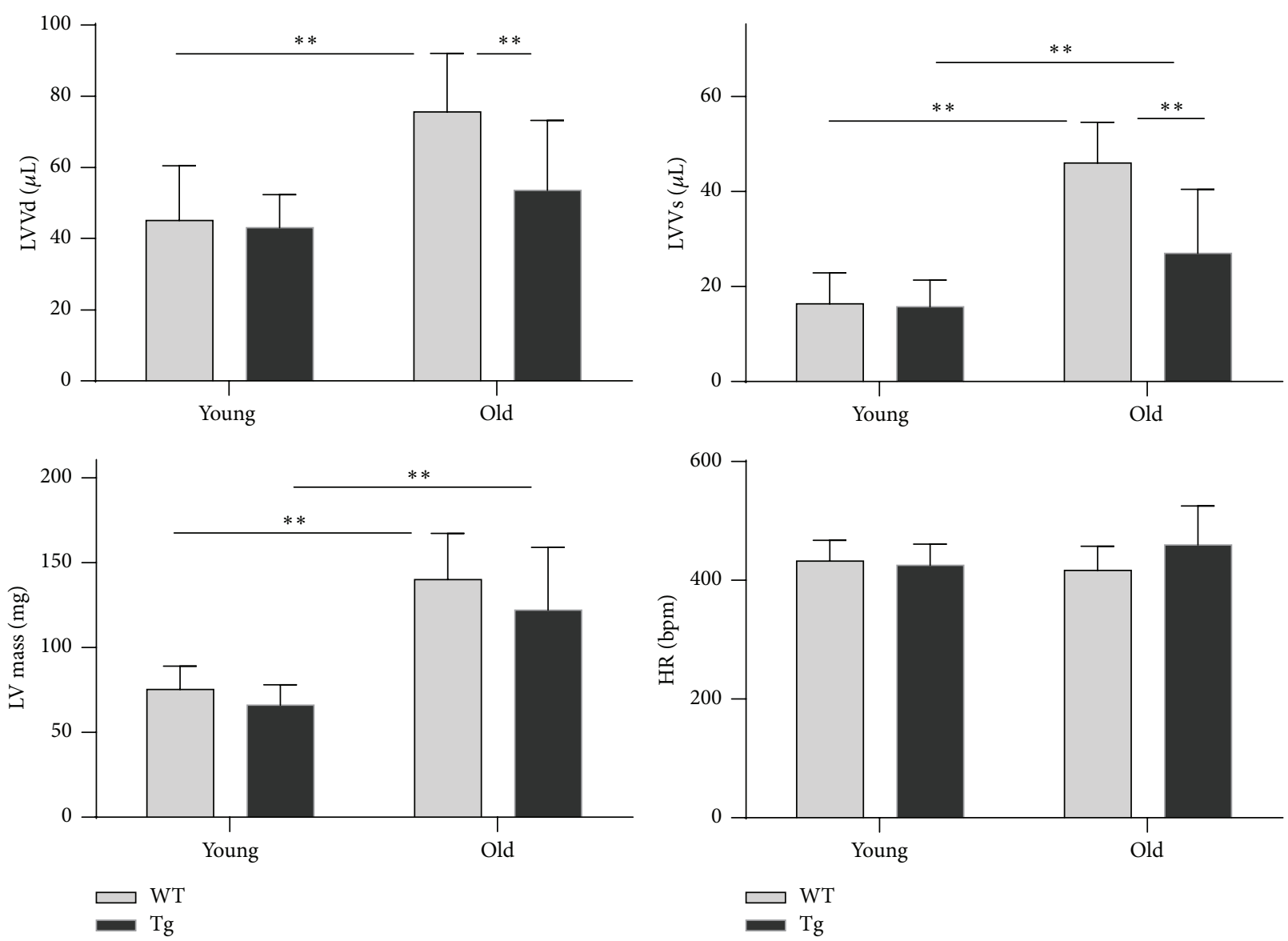

(e)

FIGURE 1: HSP27 alleviates the aging-induced decline of cardiac function. (a) Expression of HSP27 in the hearts of HSP27 Tg mice. The hearts were collected from adult WT and Tg mice. Protein extracts were prepared for immunoblot analysis against HSP27. The blots against GAPDH served as loading controls. Note that the primary antibody against HSP27 had no cross-reaction with murine homologue of HSP27. $n=3$ per group. (b) Representative images of pulse doppler of the bicuspid valve echocardiography. $n=6,9,3$, and 14 in young WT, young Tg, old WT, and old Tg groups, respectively. (c) E/A ratios. ${ }^{*} P<0.05$ and ${ }^{* *} P<0.01 . n=6,9,3$, and 14 in young WT, young Tg, old WT, and old Tg groups, respectively. (d) Representative images of M-mode tracing of echocardiography. $n=10,10,7$, and 38 in young WT, young Tg, old WT, and old Tg groups, respectively. (e) EF, FS, LVVd, and LVVs values. ${ }^{* *} P<0.01 . n=10,10,7$, and 38 in young WT, young Tg, old WT, and old Tg groups, respectively.

as described in our previous studies $[9,18]$. Briefly, cellular proteins were prepared from ventricular samples, separated by $10 \%$ SDS-PAGE, and transferred onto Immobilon-P membranes (Millipore). The membranes were probed with appropriate primary antibodies, followed by incubation with peroxidase-conjugated secondary antibodies. The signals were detected by enhanced Pierce chemiluminescence. The blots for GAPDH served as a loading control. The signals were quantified by scanning densitometry, and the results from each experimental group were expressed as relative integrated intensity compared with that of controls.

2.9. Statistical Analysis. Data are presented as mean \pm standard deviation. Groups were compared using Student's twotailed unpaired $t$-test or one-way analysis of variance followed by a post hoc procedure (Tukey's test) for multiple range, as appropriate, with SPSS 13.0 software (SPSS Inc., Chicago, IL). Statistical significance was set at $P<0.05$.

\section{Results}

3.1. HSP27 Alleviates the Aging-Induced Decline in Cardiac Function. To evaluate the roles of HSP27 in cardiac aging, we generated Tg mice expressing the human HSP27 gene. Successful expression of the transgene was confirmed by Western blot analysis, which demonstrated abundant expression of HSP27 in Tg mouse hearts, but not in WT controls (the antibody against HSP27 did not cross-react with murine HSP25) (Figure 1(a)).

The decline in cardiac function is a hallmark of cardiac aging [20]. Therefore, we examined cardiac performance using two-dimensional echocardiography in old (24-monthold) and young (2-month-old) mice. Cardiac diastolic function was reflected by the ratio of peak early to late diastolic filling velocity (E/A ratio). Old WT mice demonstrated a significant decrease in the E/A ratio by $37.5 \%$ compared with young WT mice $(P<0.01)$ (Figures $1(\mathrm{~b})$ and $1(\mathrm{c}))$. Furthermore, 


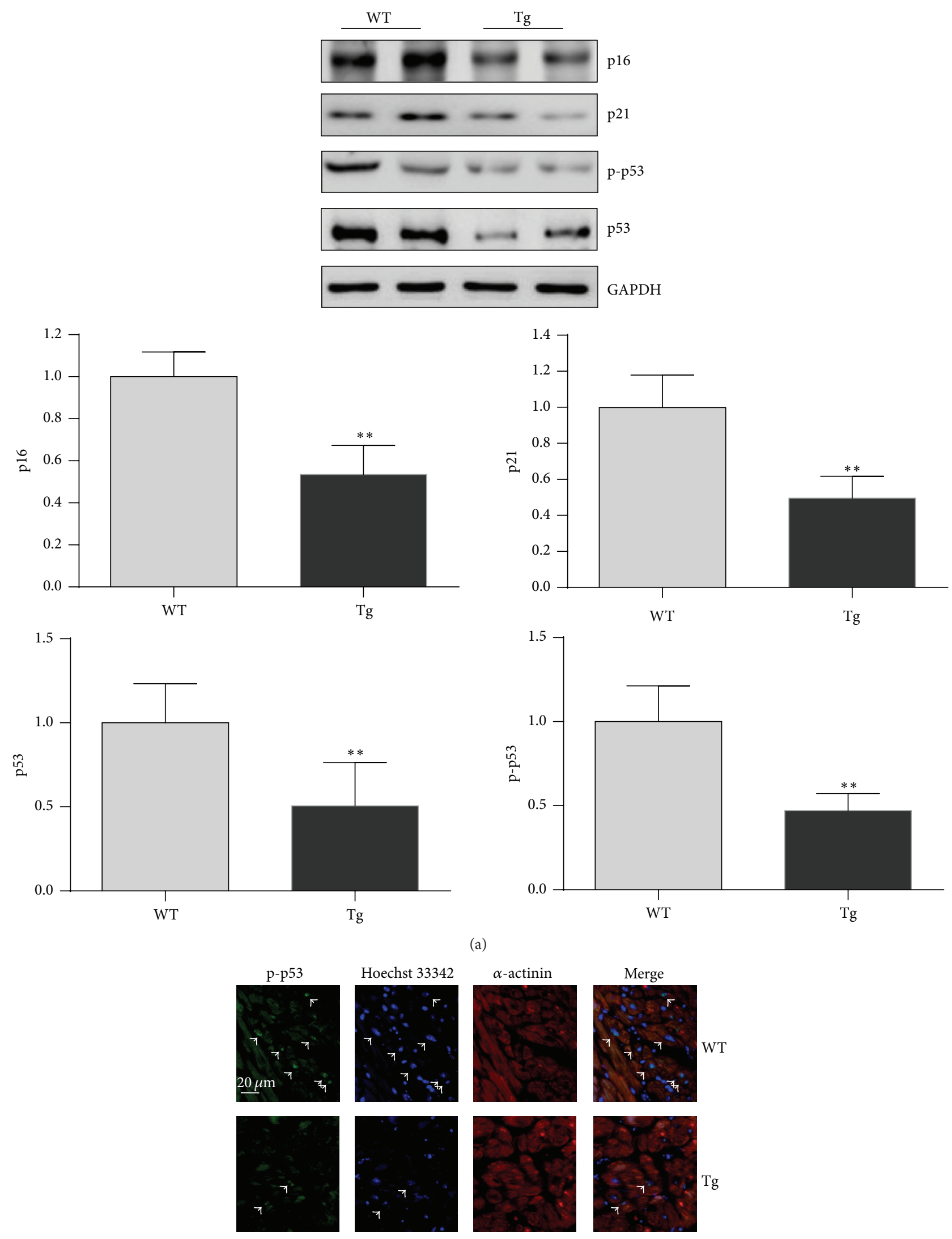

(b)

FIGURE 2: HSP27 decreases the expression of p16 and p53 in hearts of old mice. The hearts were collected from 24-month-old mice. Protein extracts were prepared for immunoblot analysis against p16 and p53. The blots against GAPDH served as loading controls. ${ }^{* *} P<0.01$ versus WT controls. $n=3$ per group. 

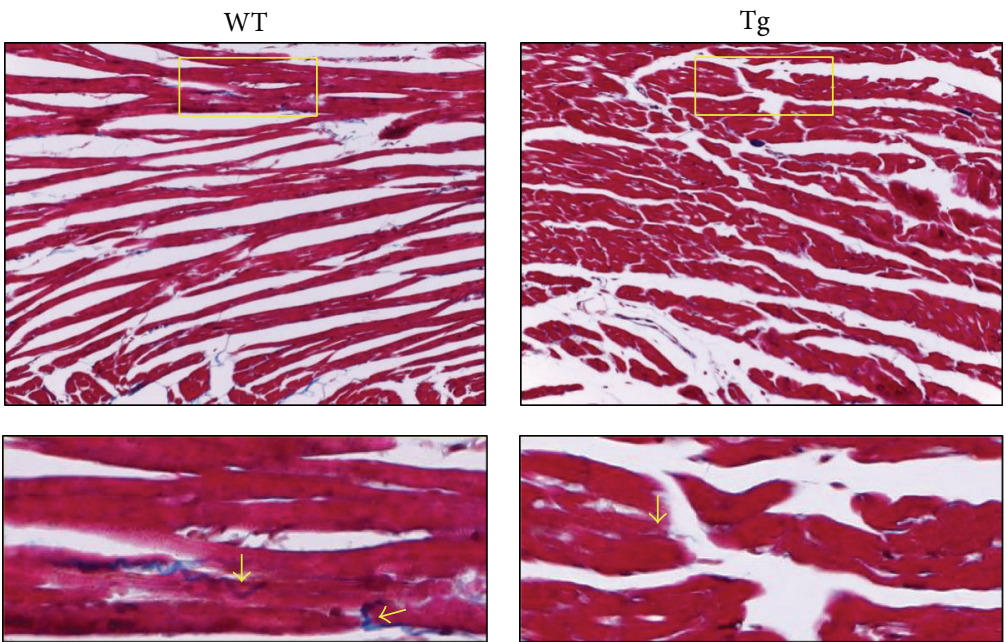

(a)

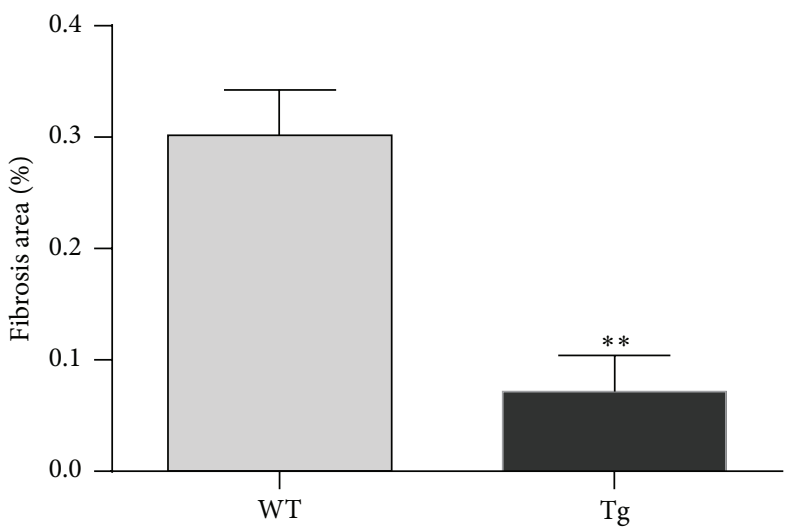

(b)

FIGURE 3: HSP27 reduces fibrosis deposition in interstitial myocardium of old mice. The hearts collected from 24-month-old mice were prepared for paraffin-embedded sectioning. Masson's trichrome staining was performed on the sections to evaluate the fibrosis (indicated by arrows). Higher magnification images of the boxed areas are shown in the down panels. Representative images from three independent experiments are shown (a). The quantification of fibrosis was shown (b). ${ }^{* *} P<0.01$ versus WT controls. $n=3$ per group.

the E/A ratio was decreased by $15.9 \%$ in old $\mathrm{Tg}$ mice compared with young $\mathrm{Tg}$ controls $(P<0.05)$. However, the aging-induced decrease in the E/A ratio was significantly attenuated by $37.4 \%$ in $\mathrm{Tg}$ mice compared with WT mice $(P<0.05)$.

Cardiac systolic function was reflected by ejection fraction (EF), fraction shortening (FS), and left ventricular (LV) volumes at end-diastolic and end-systolic phases (LVVd and LVVs, resp.). Old WT mice demonstrated significant decreases in EF (40.0\%) and FS (45.9\%) and increases in LVVd (67.4\%) and LVVs (181.0\%) compared with young WT mice $(P<0.01)$ (Figures $1(\mathrm{~d})$ and $1(\mathrm{e}))$. By contrast, EF, FS, and LVVd remained normal in old Tg mice compared with young Tg controls. Compared with old WT mice, EF and FS were significantly increased and LVVd and LVVs were significantly decreased in old Tg mice $(P<0.01)$.

The LV mass was significantly higher in both old WT mice $(85.7 \%)$ and old Tg mice $(84.7 \%)$ than in their genotypematched young controls $(P<0.01)$. However, the LV mass did not differ between old WT and old Tg mice. The heart rate did not differ between the young and old groups (Figure 1(e)).

3.2. HSP27 Decreases the Levels of Aging Markers ( $p 53$ and p16) in the Myocardium of Old Mice. The cell cycle regulators p16 and p53 play important roles in cellular senescence and therefore serve as aging markers [21, 22]. Therefore, the expression levels of p16 and p53 were examined in hearts of old mice. The results of immunoblot analysis are shown in Figure 2(a). Levels of p16 and p53 were significantly reduced by $46.5 \%$ and $49.4 \%$, respectively, in hearts of old Tg mice compared with hearts of old WT mice $(P<0.01)$.

Expression of p21 is regulated by $\mathrm{p} 53$. Hearts of old $\mathrm{Tg}$ mice demonstrated a significantly lower level of p21 than hearts of old WT mice $(P<0.01)$ (Figure $2(a))$.

To determine the activation of $\mathrm{p} 53$, we examined the phosphorylation level of p53 at Ser15 (p-p53). The p-p53 level was significantly lower in hearts of old $\mathrm{Tg}$ mice $(53.0 \%)$ than in hearts of old WT mice $(P<0.01)$ (Figure $2(\mathrm{a}))$. 


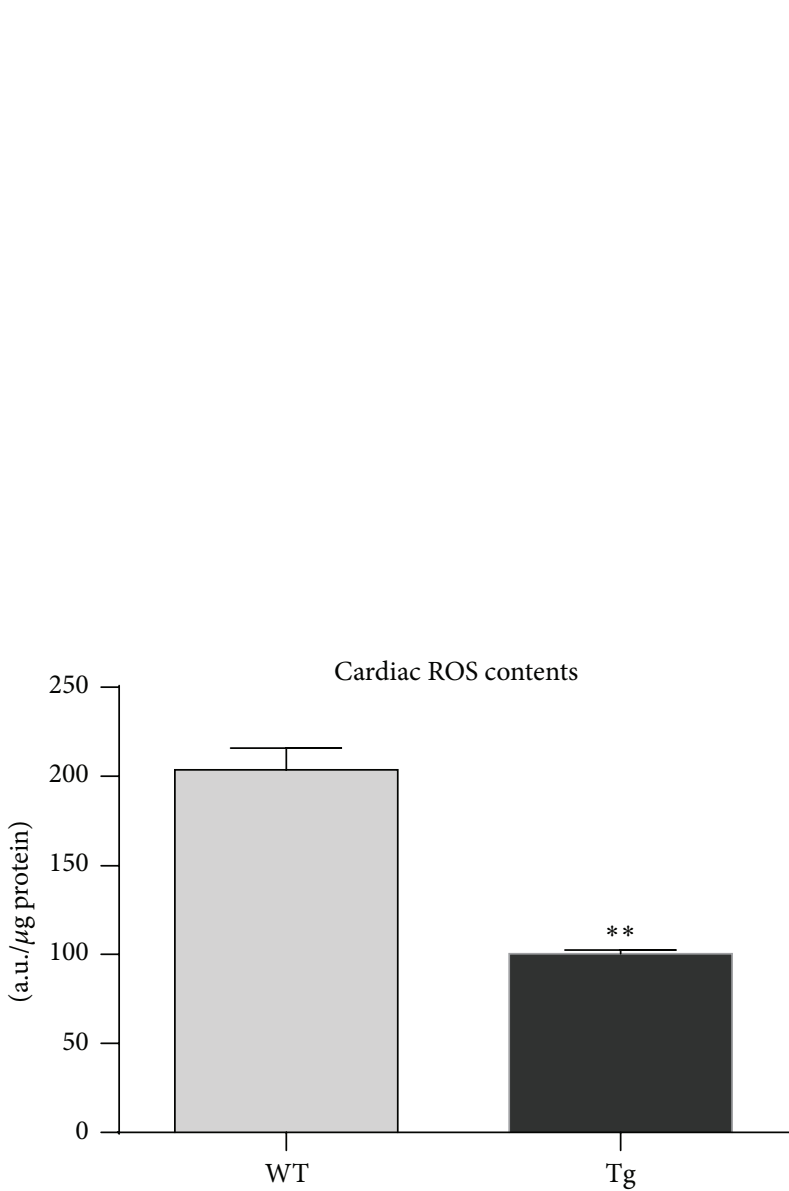

(a)
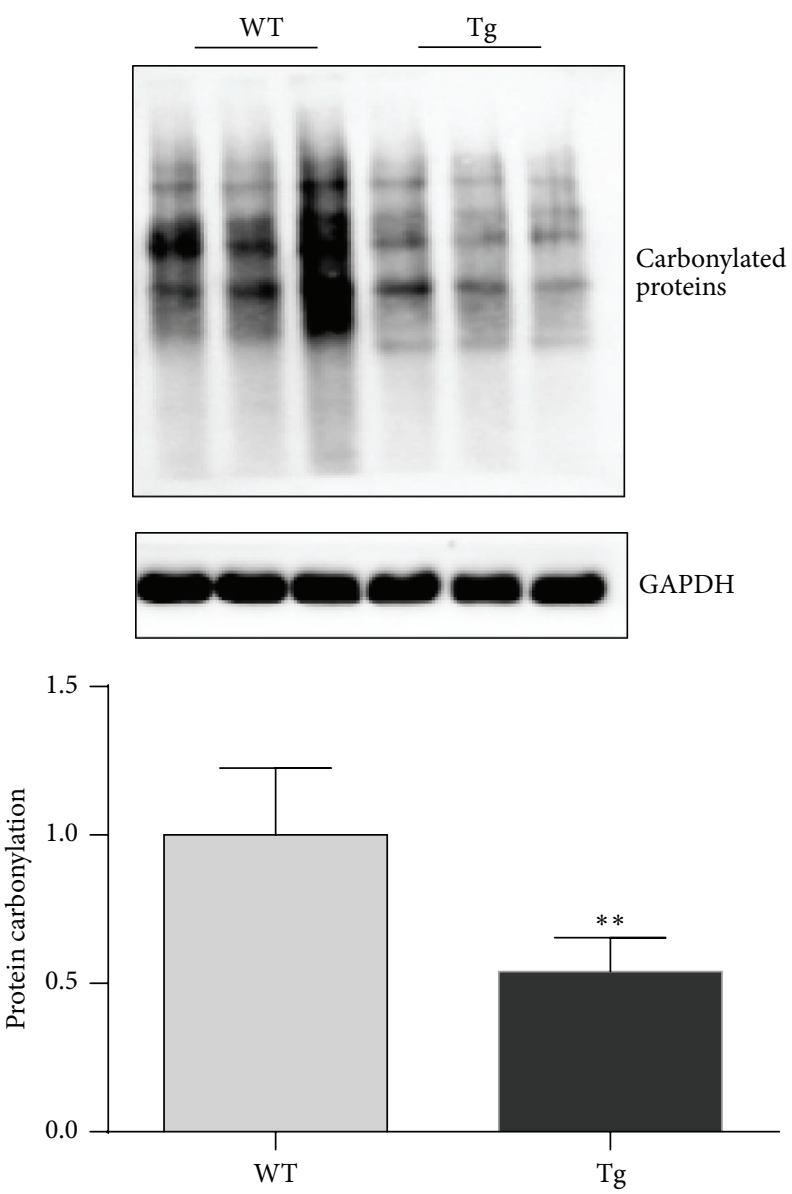

(b)

FIGURE 4: HSP27 decreases ROS contents in the hearts of old mice. The hearts were collected from 24-month-old mice. DCFH assay was performed to evaluate intracellular ROS contents. The relative contents of ROS were expressed as the arbitrary units per $\mu \mathrm{g}$ protein. ${ }^{* *} P<0.01$ versus WT controls. $n=3$ per group.

To determine whether the activation of p53 occurred in cardiomyocytes, we performed fluorescence immunohistochemistry of cardiac tissues. Positive staining of p-p53 was localized in the nuclei of cardiomyocytes in the hearts of both old WT and old Tg mice (Figure 2(b)).

\subsection{HSP27 Attenuates Fibrosis in the Interstitial Myocardium} of Old Mice. Compromised diastolic function is associated with myocardial interstitial fibrosis [23]. We examined myocardial fibrosis in old mice using Masson's trichrome staining. Obvious fibrosis was observed in the myocardial interstitium of old WT mice (indicated by arrows) (Figures $3(\mathrm{a})$ and $3(\mathrm{~b})$ ). By contrast, there was significantly less fibrosis in old Tg mouse hearts than in old WT mouse hearts. Fibrosis was significantly reduced by $76.2 \%$ in old $\mathrm{Tg}$ mouse hearts compared with old WT mouse hearts $(P<0.01)$.

3.4. HSP27 Decreases the ROS Content in the Myocardium of Old Mice. ROS are one of the critical players in the development of cardiac aging [1]. To determine whether ROS are involved in the protective effect of HSP27 against the aging-induced decline in cardiac function, we measured ROS contents in the myocardium of old mice. The ROS content was 2038.6 and 752.4 units in old WT and old Tg mouse hearts, respectively (Figure 4(a)). Therefore, hearts of old $\mathrm{Tg}$ mice demonstrated a significantly lower ROS content (by 63.1\%) than old WT mouse hearts $(P<0.01)$.

Protein carbonylation was also examined in hearts of old mice. Protein carbonylation was significantly lower in hearts of old Tg mice $(46.1 \%)$ than in hearts of WT mice $(P<0.01)$ (Figure 4(b)).

3.5. HSP27 Decreases the Accumulation of LC3-II and p62 in Old Hearts. Activation of mitochondrial autophagy (mitophagy) alleviates cardiac aging [24]. We first examined LC3 conversion and p62 protein degradation, two widely used markers of autophagic flux [25], in hearts by immunoblot analysis. The level of LC3-II was significantly decreased by $38.7 \%$ in old $\mathrm{Tg}$ mouse hearts compared with old WT mouse hearts $(P<0.01)$ (Figure 5(a)). Immunostaining of LC3-II confirmed the observation made in immunoblot analysis (Figure 5(b)). By contrast, the p62 protein level was reduced by $59.0 \%$ in old $\mathrm{Tg}$ mouse hearts compared with old WT mouse hearts $(P<0.01)$. These data 

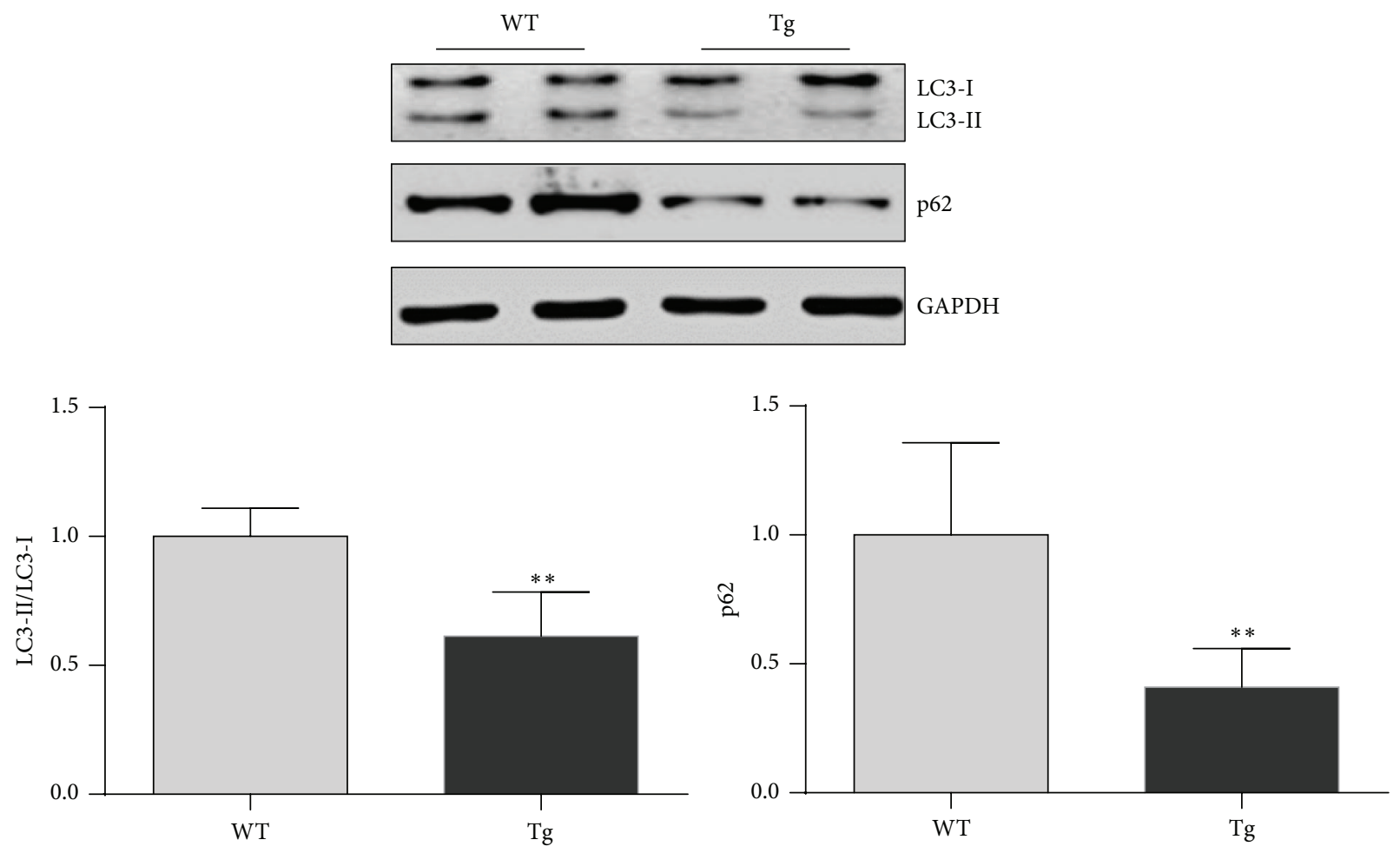

(a)

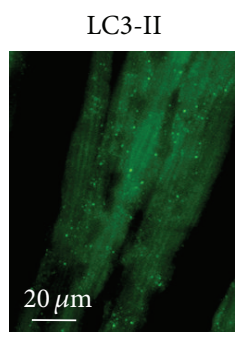

Hoechst 33342
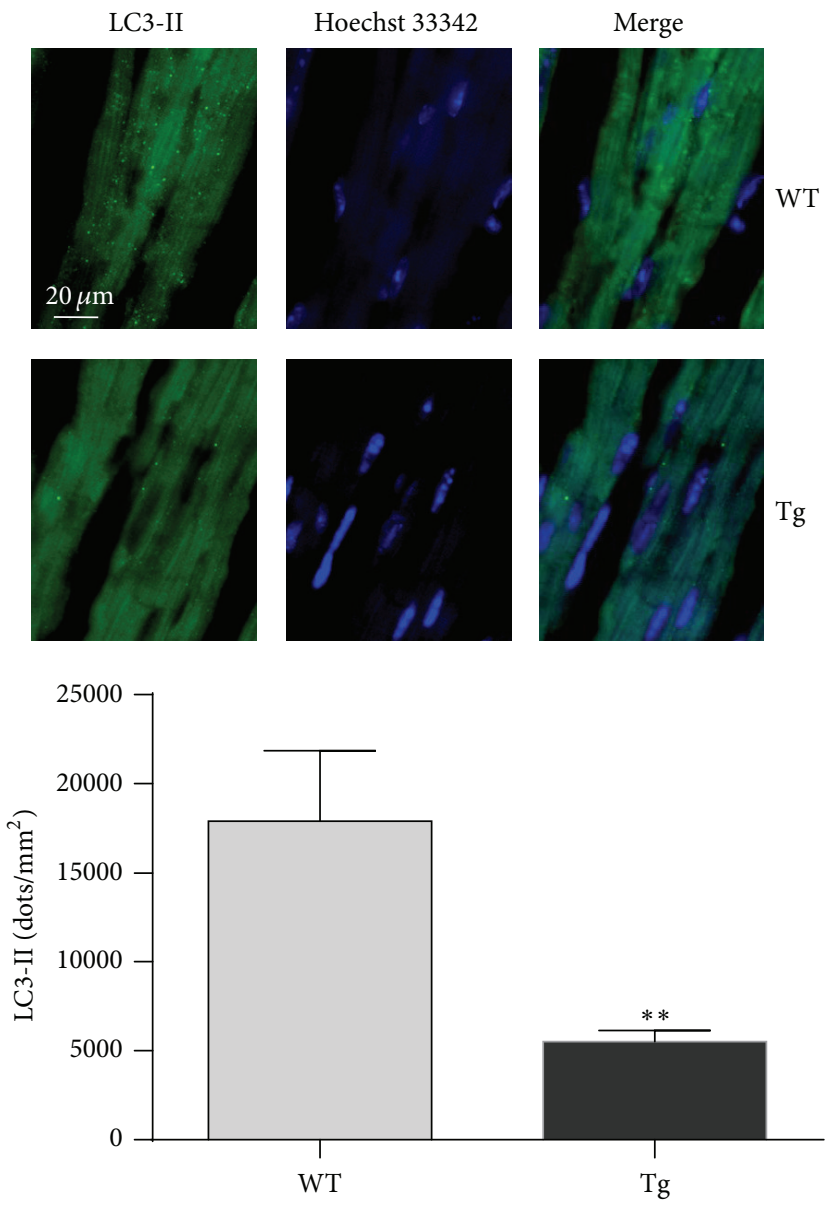

(b)

Figure 5: Continued. 

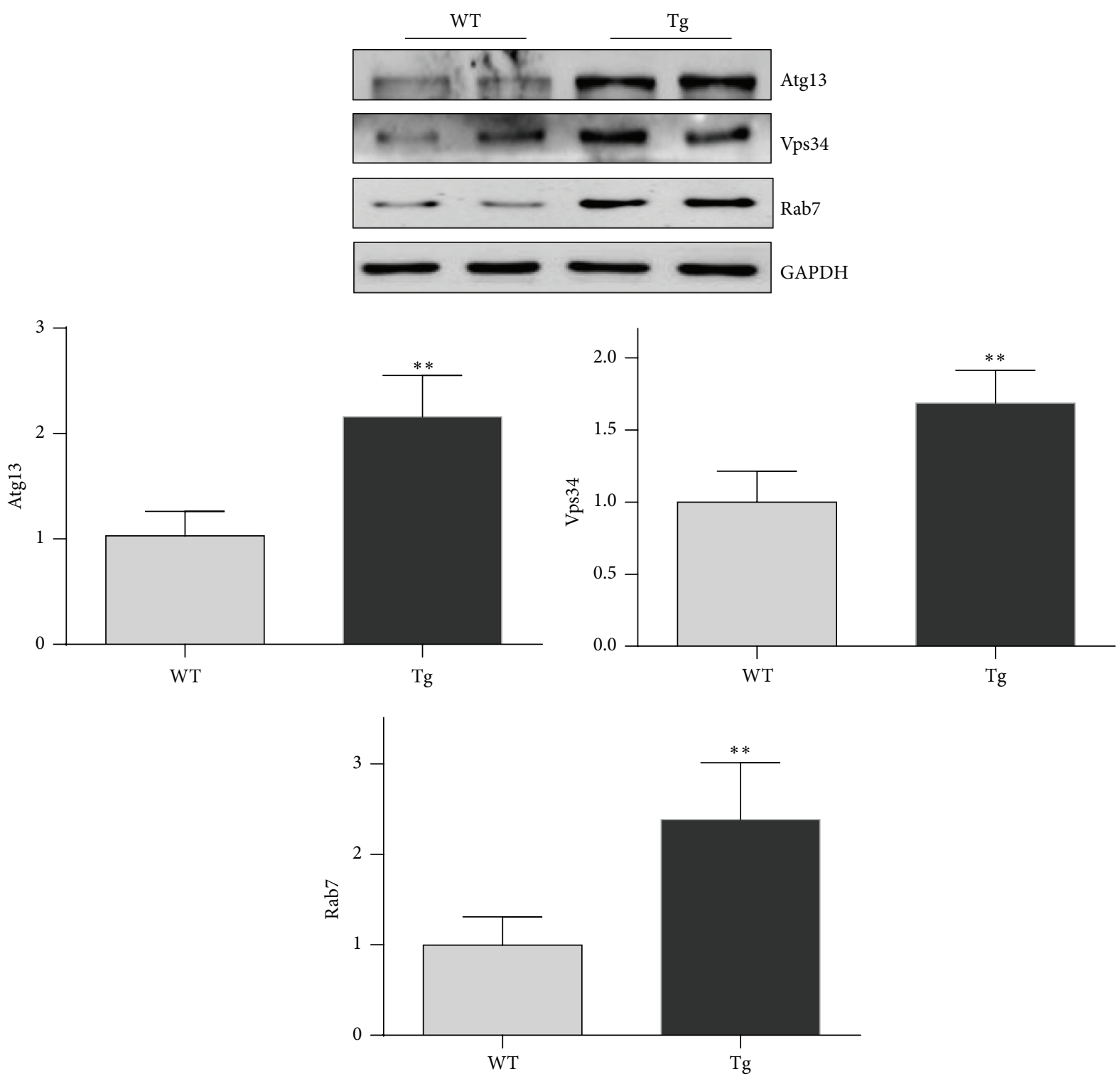

(c)

FIGURE 5: HSP27 increases autophagic markers in the hearts of old mice. The hearts were collected from 24-month-old mice. Protein extracts were prepared for immunoblot analysis against LC3 and p62 (a) and against Atg 13, Vps34, and Rab7 (b). The blots against GAPDH served as loading controls. ${ }^{* *} P<0.01$ versus WT controls. $n=3$ per group.

suggest that autophagic flux was significantly higher in old $\mathrm{Tg}$ mouse hearts than in old WT mouse hearts.

Atg13, Vps34, and Rab7 play important roles in the formation and maturation of autophagosomes [26-28]. Hearts of old Tg mice demonstrated significant increases in the levels of Atg13 (112.7\%), Vps34 (68.5\%), and Rab7 (138.4\%) compared with hearts of old WT mice $(P<0.01)$ (Figure 5(c)).

3.6. HSP27 Increases PINK1 and Parkin Expression in Old Hearts. PINK1 and Parkin are important for mitophagy induction [29]. The levels of PINK1 and Parkin were increased by $57.1 \%$ and $60.5 \%$, respectively, in hearts of old $\mathrm{Tg}$ mice compared with hearts of old WT mice $(P<0.01)$ (Figure 6). The mitophagy receptors BNIP3 and Nix were also examined by immunoblot analysis. The results demonstrated a significantly higher level of BNIP3 in hearts of old Tg mice compared with hearts of old WT mice $(P<0.05)$. The Nix levels were comparable between the two genotypes.

\subsection{HSP27 Reduces the Content of Ubiquitin-Conjugated} Proteins. Autophagy is an important approach for the degradation of ubiquitin-conjugated proteins and damaged organelles including mitochondria [30]. We examined the levels of ubiquitin-conjugated proteins. Hearts of old Tg mice exhibited significantly lower levels of protein ubiquitination (76.2\%) than hearts of old WT mice $(P<0.01)$ (Figure $7(\mathrm{a}))$. As protein loading controls, the SDS-PAGE gel was stained 


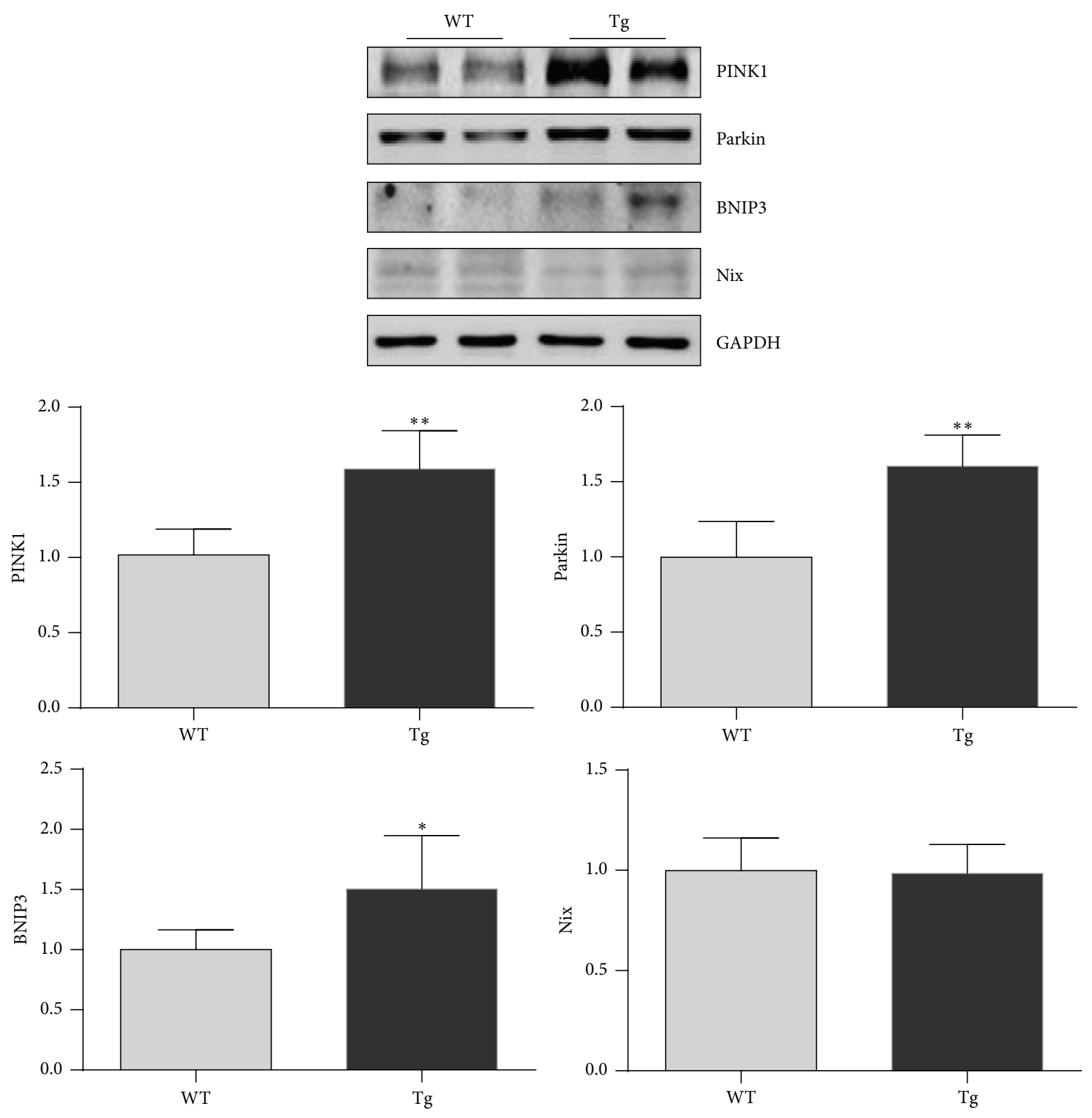

FIgURE 6: HSP27 increases PINK1 and Parkin expression in the hearts of old mice. The hearts were collected from 24-month-old mice. Protein extracts were prepared for immunoblot analysis against PINK1 and Parkin. The blots against GAPDH served as loading controls. ${ }^{*} P<0.05$ and ${ }^{* *} P<0.01$ versus WT controls. $n=3$ per group.

with Coomassie blue (Figure 7(b)) and the blot was probed for GAPDH (Figure 7(b)).

\section{Discussion}

The significant finding of this study is that cardiac-specific expression of HSP27 attenuated the aging-induced decline in cardiac function of mice. Reduced expression of cardiac aging markers, less interstitial fibrosis, and lower levels of ROS and ubiquitin-conjugated proteins were observed in old Tg mouse hearts compared with old WT mouse hearts. Moreover, old Tg mouse hearts showed significantly decreased accumulation of LC3-II and p62 proteins and increased expression of Atg13, Vps34, Rab7, PINK1, and Parkin compared with old WT mouse hearts. Taken together, these results suggest that HSP27 alleviates cardiac aging and that this action involves antioxidation and mitophagy activation.

Cardiac aging is associated with compromised cardiac function. In humans, the LV early diastolic filling rate progressively slows after the age of 20 years, and therefore by 80 years of age the rate is reduced, on average, by up to $50 \%$ [20]. EF, a parameter indicative of LV systolic function, can decrease by more than $20 \%$ in healthy old individuals [20]. Consistent with this, aged animals also demonstrate 


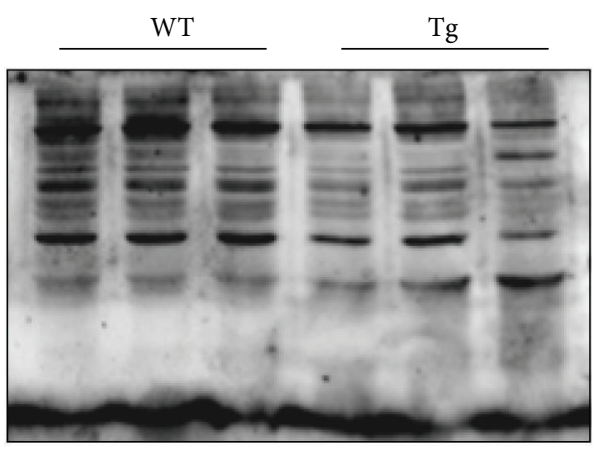

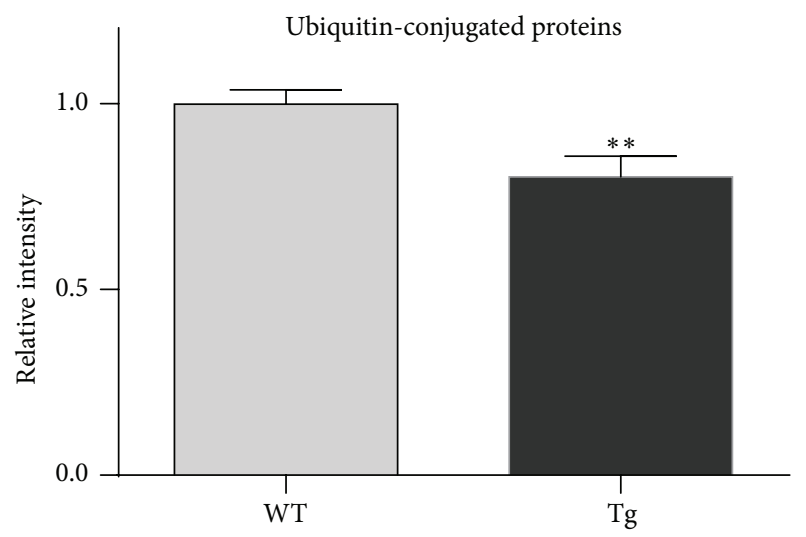

(a)

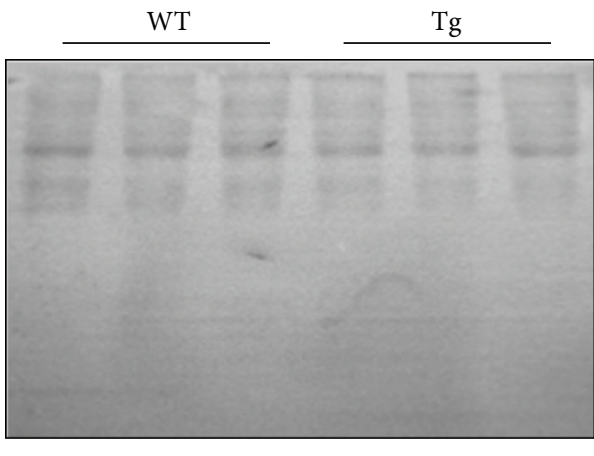

(b)

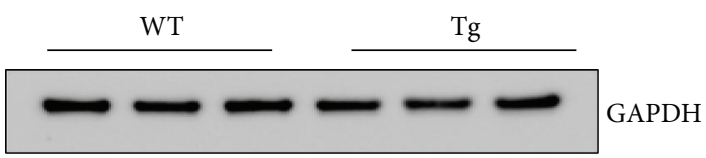

(c)

FIGURE 7: HSP27 decreases the levels of ubiquitin-conjugated protein in the hearts of old mice. The hearts were collected from 24-month-old mice. Protein extracts were prepared for immunoblot analysis against ubiquitin (a). The Coomassie blue-stained gel served as loading control (b). ${ }^{* *} P<0.01$ versus WT controls. $n=3$ per group.

an impairment in cardiac function $[1,31]$. In this study, cardiac systolic and diastolic functions were reduced and LV diameters were increased in 24-month-old WT mice. However, the aging-induced decline in cardiac function and the enlargement of LV diameter were attenuated in HSP27 $\mathrm{Tg}$ mice. These results suggest that HSP27 overexpression improves the aging-induced impairment of cardiac performance.

While senescence is reflected by phenotypic changes in functions and structures, the molecular mechanism underlying cellular senescence involves p16- and p53-mediated pathways [32]. p16 and p53, two important tumor suppressors, mediate senescence through inhibiting the action of cyclin-dependent kinases, which in turn leads to G1 arrest of the cell cycle [32]. Indeed, an inverse relationship between the expression levels of p16/p53 and senescence was well demonstrated by studies of both humans and animals $[32,33]$. Therefore, $\mathrm{p} 16$ and $\mathrm{p} 53$ are indicated to be signatures of in vivo senescence $[33,34]$. In this study, significantly lower levels of p16 and p53 expression were detected in old Tg mouse hearts compared with old WT mouse hearts, suggesting that aging-induced decline of cardiac function was alleviated by HSP27 overexpression.

The well-known free radical theory of aging, first proposed by Harman in 1956, posits that intracellular production of ROS is the major determinant of lifespan $[1,35]$. Indeed, antioxidative interventions, such as overexpression of mitochondrial catalase, significantly improve cardiac systolic and diastolic functions and decrease protein oxidative damage in aged mice [36]. Moreover, antioxidation reduces the expression of p16 and p53 [37, 38]. In this study, old Tg mouse hearts exhibited significantly lower levels of ROS and protein carbonylation than old WT mouse hearts. Supporting this, we and others revealed that HSP27 possesses an antioxidative capacity in myocytes and other cells $[13,39]$. Taken together, our results suggest that antioxidation was involved in the protective effect of HSP27 against aging-induced decline of cardiac function.

Autophagy is an evolutionarily conserved process involved in the degradation of damaged proteins, mitochondria, and other organelles [40]. The intracellular accumulation of damaged macromolecules and organelles is a feature 
common to all aged cells [41]. The postmitotic nature of cardiomyocytes makes these cells especially prone to the accumulation of macromolecular and organellar oxidative damage over the lifetime, which means that more autophagic activation is required to clean up this harmful cellular "garbage" in order to maintain cardiomyocyte homeostasis [1]. Unfortunately, autophagic activity in cardiomyocytes declines with age, while impaired autophagy has multiple consequences including decreased contractile function and increased arrhythmia risk [42, 43]. Evidence suggests that stimulation of autophagy via genetic deletion of endothelin receptor A rescues the aging-induced impairment of cardiac contractile dysfunction [43]. In this study, we observed increased activation of autophagy, which was reflected by decreased accumulation of LC3 and p62, in old HSP27 Tg mouse hearts compared with old WT mouse hearts. Collectively, these data suggest that autophagic activation is involved in the protective effect of HSP27 against cardiac aging.

Mitophagy refers to selective autophagy for mitochondrial degradation. Mitochondria are both the main source of ROS generation and targets of ROS attack. Due to the high reliance of cardiomyocytes on mitochondrial energy metabolism, the heart is exposed to a high burden of ROS throughout the lifetime [1]. Activation of PINK1 and Parkin plays a critical role in turnover of damaged mitochondria through mitophagy. PINK1 kinase activity and its mitochondrial localization are prerequisites for inducing translocation of the E3 ligase Parkin to depolarized mitochondria. Subsequently, Parkin mediates the formation of two distinct polyubiquitin chains to recruit autophagy receptors [44]. Thus, PINK1 signals mitochondrial dysfunction to Parkin, and Parkin promotes the elimination of damaged mitochondria. Moreover, p53 inhibits Parkin-mediated mitophagy [45]. In this study, we observed significant increases in PINK1 and Parkin levels in old Tg mouse hearts compared with old WT mouse hearts. By contrast, a lower level of p53 was detected in old Tg mouse hearts compared with old WT mouse hearts. Taken together, our data suggest that activation of mitophagy contributes to the alleviation of cardiac aging by HSP27.

In summary, our data provide functional links between HSP27 expression and improvement of the aging-induced decline in cardiac function, which suggests that management of HSP27 expression serves as a potential intervention for alleviation of cardiac aging.

\section{Competing Interests}

The authors declare that there are no competing interests regarding the publication of this paper.

\section{Acknowledgments}

This work was supported by the National Natural Science Foundation of China (81571290, 81571378, 81370260, 81371450, and 81170321), by Jiangsu Province's Outstanding Medical Academic Leader Program (LJ201124), by Project Funded by the Priority Academic Program Development of Jiangsu Higher Education Institutions (PAPD), and by a grant from Collaborative Innovation Center for Cardiovascular Disease Translational Medicine.

\section{References}

[1] S. E. Wohlgemuth, R. Calvani, and E. Marzetti, "The interplay between autophagy and mitochondrial dysfunction in oxidative stress-induced cardiac aging and pathology," Journal of Molecular and Cellular Cardiology, vol. 71, pp. 62-70, 2014.

[2] D.-F. Dai, T. Chen, S. C. Johnson, H. Szeto, and P. S. Rabinovitch, "Cardiac aging: from molecular mechanisms to significance in human health and disease," Antioxidants and Redox Signaling, vol. 16, no. 12, pp. 1492-1536, 2012.

[3] Y. A. Chiao and P. S. Rabinovitch, "The aging heart," Cold Spring Harbor Perspectives in Medicine, vol. 5, no. 9, Article ID a025148, 2015.

[4] E. K. Quarles, D.-F. Dai, A. Tocchi, N. Basisty, L. Gitari, and P. S. Rabinovitch, "Quality control systems in cardiac aging," Ageing Research Reviews, vol. 23, part A, pp. 101-115, 2015.

[5] S. E. Schriner, N. J. Linford, G. M. Martin et al., "Extension of murine life span by overexpression of catalase targeted to mitochondria," Science, vol. 308, no. 5730, pp. 1909-1911, 2005.

[6] D.-F. Dai and P. S. Rabinovitch, "Cardiac aging in mice and humans: the role of mitochondrial oxidative stress," Trends in Cardiovascular Medicine, vol. 19, no. 7, pp. 213-220, 2009.

[7] J. J. Lemasters, "Selective mitochondrial autophagy, or mitophagy, as a targeted defense against oxidative stress, mitochondrial dysfunction, and aging," Rejuvenation Research, vol. 8, no. 1, pp. 3-5, 2005.

[8] Y. Ikeda, S. Sciarretta, N. Nagarajan et al., "New insights into the role of mitochondrial dynamics and autophagy during oxidative stress and aging in the heart," Oxidative Medicine and Cellular Longevity, vol. 2014, Article ID 210934, 13 pages, 2014.

[9] W. You, X. Min, X. Zhang et al., "Cardiac-specific expression of heat shock protein 27 attenuated endotoxin-induced cardiac dysfunction and mortality in mice through a PI3K/Aktdependent mechanism," Shock, vol. 32, no. 1, pp. 108-117, 2009.

[10] I. J. Benjamin and D. R. McMillan, "Stress (heat shock) proteins: molecular chaperones in cardiovascular biology and disease," Circulation Research, vol. 83, no. 2, pp. 117-132, 1998.

[11] J. M. Hollander, J. L. Martin, D. D. Belke et al., "Overexpression of wild-type heat shock protein 27 and a nonphosphorylatable heat shock protein 27 mutant protects against ischemia/reperfusion injury in a transgenic mouse model," Circulation, vol. 110, no. 23, pp. 3544-3552, 2004.

[12] L. M. McGinley, J. McMahon, A. Stocca et al., "Mesenchymal stem cell survival in the infarcted heart is enhanced by lentivirus vector-mediated heat shock protein 27 expression," Human Gene Therapy, vol. 24, no. 10, pp. 840-851, 2013.

[13] L. Liu, X. Zhang, B. Qian et al., "Over-expression of heat shock protein 27 attenuates doxorubicin-induced cardiac dysfunction in mice," European Journal of Heart Failure, vol. 9, no. 8, pp. 762769, 2007.

[14] P. J. Vernon and D. Tang, "Eat-me: autophagy, phagocytosis, and reactive oxygen species signaling," Antioxidants and Redox Signaling, vol. 18, no. 6, pp. 677-691, 2013.

[15] P. Mehlen, C. Kretz-Remy, X. Préville, and A.-P. Arrigo, "Human hsp27, Drosophila hsp27 and human $\alpha \mathrm{B}$-crystallin expression-mediated increase in glutathione is essential for the protective activity of these proteins against TNF $\alpha$-induced cell death," The EMBO Journal, vol. 15, no. 11, pp. 2695-2706, 1996. 
[16] P. Mehlen, X. Preville, P. Chareyron, J. Briolay, R. Klemenz, and A.-P. Arrigo, "Constitutive expression of human hsp27, Drosophila hsp27, or human $\alpha \mathrm{B}$-crystallin confers resistance to TNF- and oxidative stress-induced cytotoxicity in stably transfected murine L929 fibroblasts," Journal of Immunology, vol. 154, no. 1, pp. 363-374, 1995.

[17] X. Préville, F. Salvemini, S. Giraud et al., "Mammalian small stress proteins protect against oxidative stress through their ability to increase glucose-6-phosphate dehydrogenase activity and by maintaining optimal cellular detoxifying machinery," Experimental Cell Research, vol. 247, no. 1, pp. 61-78, 1999.

[18] J. Li, Y. Zhang, C. Li et al., "HSPA12B attenuates cardiac dysfunction and remodelling after myocardial infarction through an eNOS-dependent mechanism," Cardiovascular Research, vol. 99, no. 4, pp. 674-684, 2013.

[19] L.-J. Yan, N. S. Rajasekaran, S. Sathyanarayanan, and I. J. Benjamin, "Mouse HSF1 disruption perturbs redox state and increases mitochondrial oxidative stress in kidney," Antioxidants and Redox Signaling, vol. 7, no. 3-4, pp. 465-471, 2005.

[20] E. G. Lakatta and D. Levy, "Arterial and cardiac aging: major shareholders in cardiovascular disease enterprises. Part II. The aging heart in health: links to heart disease," Circulation, vol. 107, no. 2, pp. 346-354, 2003.

[21] N. Hariharan, P. Quijada, S. Mohsin et al., "Nucleostemin rejuvenates cardiac progenitor cells and antagonizes myocardial aging," Journal of the American College of Cardiology, vol. 65, no. 2, pp. 133-147, 2015.

[22] Y. Hua, T. J. Robinson, Y. Cao, G.-P. Shi, J. Ren, and S. Nair, "Cathepsin K knockout alleviates aging-induced cardiac dysfunction," Aging Cell, vol. 14, no. 3, pp. 345-351, 2015.

[23] A. L. Reed, A. Tanaka, D. Sorescu et al., "Diastolic dysfunction is associated with cardiac fibrosis in the senescence-accelerated mouse," American Journal of Physiology-Heart and Circulatory Physiology, vol. 301, no. 3, pp. H824-H831, 2011.

[24] D. Dutta, R. Calvani, R. Bernabei, C. Leeuwenburgh, and E. Marzetti, "Contribution of impaired mitochondrial autophagy to cardiac aging: mechanisms and therapeutic opportunities," Circulation Research, vol. 110, no. 8, pp. 1125-1138, 2012.

[25] L. Vucicevic, M. Misirkic-Marjanovic, V. Paunovic et al., "Autophagy inhibition uncovers the neurotoxic action of the antipsychotic drug olanzapine," Autophagy, vol. 10, no. 12, pp. 2362-2378, 2014.

[26] F. Li and R. D. Vierstra, "Arabidopsis ATG11, a scaffold that links the ATG1-ATG13 kinase complex to general autophagy and selective mitophagy," Autophagy, vol. 10, no. 8, pp. 14661467, 2014.

[27] B. Pasquier, "SAR405, a PIK3C3/Vps34 inhibitor that prevents autophagy and synergizes with MTOR inhibition in tumor cells," Autophagy, vol. 11, no. 4, pp. 725-726, 2015.

[28] Z. Lu, H. Yang, M. N. Sutton et al., "ARHI (DIRAS3) induces autophagy in ovarian cancer cells by downregulating the epidermal growth factor receptor, inhibiting PI3K and Ras/MAP signaling and activating the FOXo3a-mediated induction of Rab7," Cell Death and Differentiation, vol. 21, no. 8, pp. 12751289, 2014.

[29] E. S. Vincow, G. Merrihew, R. E. Thomas et al., "The PINK1parkin pathway promotes both mitophagy and selective respiratory chain turnover in vivo," Proceedings of the National Academy of Sciences of the United States of America, vol. 110, no. 16, pp. 6400-6405, 2013.
[30] T. M. Durcan, M. Y. Tang, J. R. Pérusse et al., "USP8 regulates mitophagy by removing K6-linked ubiquitin conjugates from parkin," EMBO Journal, vol. 33, no. 21, pp. 2473-2491, 2014.

[31] Y. Zhang, S.-L. Mi, N. Hu et al., "Mitochondrial aldehyde dehydrogenase 2 accentuates aging-induced cardiac remodeling and contractile dysfunction: role of AMPK, Sirtl, and mitochondrial function," Free Radical Biology and Medicine, vol. 71, pp. 208220, 2014.

[32] H. Rayess, M. B. Wang, and E. S. Srivatsan, "Cellular senescence and tumor suppressor gene p16," International Journal of Cancer, vol. 130, no. 8, pp. 1715-1725, 2012.

[33] S. Ressler, J. Bartkova, H. Niederegger et al., "p16INK4A is a robust in vivo biomarker of cellular aging in human skin," Aging Cell, vol. 5, no. 5, pp. 379-389, 2006.

[34] N. E. Sharpless and C. J. Sherr, "Forging a signature of in vivo senescence," Nature Reviews Cancer, vol. 15, no. 7, pp. 397-408, 2015.

[35] D. Harman, "Aging: a theory based on free radical and radiation chemistry," Journal of gerontology, vol. 11, no. 3, pp. 298-300, 1956.

[36] D.-F. Dai, L. F. Santana, M. Vermulst et al., "Overexpression of catalase targeted to mitochondria attenuates murine cardiac aging," Circulation, vol. 119, no. 21, pp. 2789-2797, 2009.

[37] S. Soberanes, A. Gonzalez, D. Urich et al., "Particulate matter air pollution induces hypermethylation of the p16 promoter Via a mitochondrial ROS-JNK-DNMT1 pathway," Scientific Reports, vol. 2, article 275, 2012.

[38] Y. Shi, F. Nikulenkov, J. Zawacka-Pankau et al., "ROS-dependent activation of JNK converts p53 into an efficient inhibitor of oncogenes leading to robust apoptosis," Cell Death and Differentiation, vol. 21, no. 4, pp. 612-623, 2014.

[39] T. Rogalla, M. Ehrnsperger, X. Preville et al., "Regulation of Hsp27 oligomerization, chaperone function, and protective activity against oxidative stress/tumor necrosis factor by phosphorylation," Journal of Biological Chemistry, vol. 274, no. 27, pp. 18947-18956, 1999.

[40] R. Li, H. Ma, X. Zhang et al., "Impaired autophagosome clearance contributes to local anesthetic bupivacaine-induced myotoxicity in mouse myoblasts," Anesthesiology, vol. 122, no. 3, pp. 595-605, 2015.

[41] A. M. Cuervo, E. Bergamini, U. T. Brunk, W. Dröge, M. Ffrench, and A. Terman, "Autophagy and aging: the importance of maintaining 'clean' cells," Autophagy, vol. 1, no. 3, pp. 131-140, 2005.

[42] P.-J. Linton, M. Gurney, D. Sengstock, R. M. Mentzer, and R. A. Gottlieb, "This old heart: cardiac aging and autophagy," Journal of Molecular and Cellular Cardiology, vol. 83, pp. 44-54, 2015.

[43] A. F. Ceylan-Isik, M. Dong, Y. Zhang et al., "Cardiomyocytespecific deletion of endothelin receptor A rescues agingassociated cardiac hypertrophy and contractile dysfunction: role of autophagy," Basic Research in Cardiology, vol. 108, no. 2, article 335, 2013.

[44] M. Lazarou, D. A. Sliter, L. A. Kane et al., "The ubiquitin kinase PINK1 recruits autophagy receptors to induce mitophagy," Nature, vol. 524, no. 7565, pp. 309-314, 2015.

[45] A. Hoshino, Y. Mita, Y. Okawa et al., "Cytosolic p53 inhibits Parkin-mediated mitophagy and promotes mitochondrial dysfunction in the mouse heart," Nature Communications, vol. 4, article 2308, 2013. 


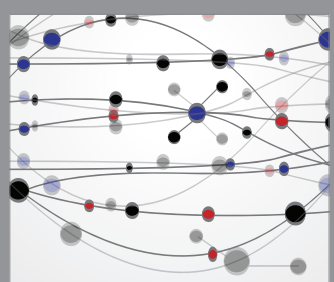

The Scientific World Journal
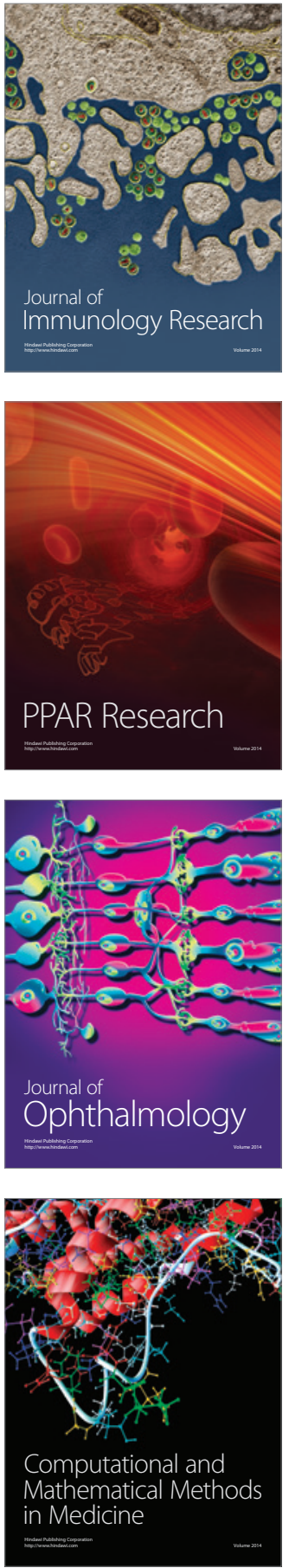

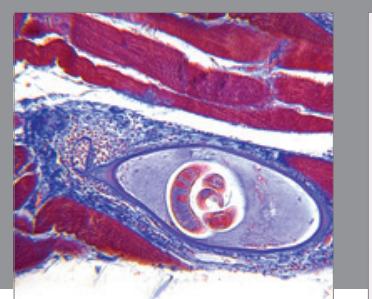

Gastroenterology Research and Practice

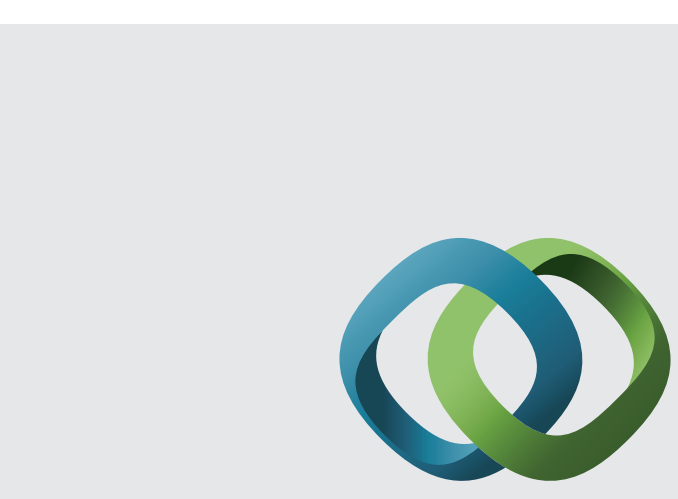

\section{Hindawi}

Submit your manuscripts at

http://www.hindawi.com
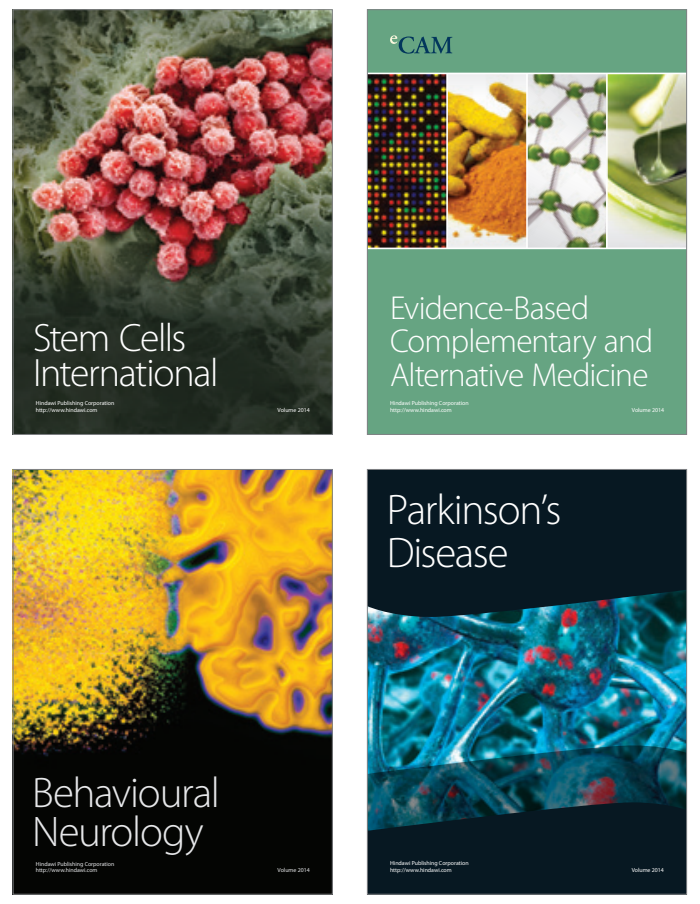
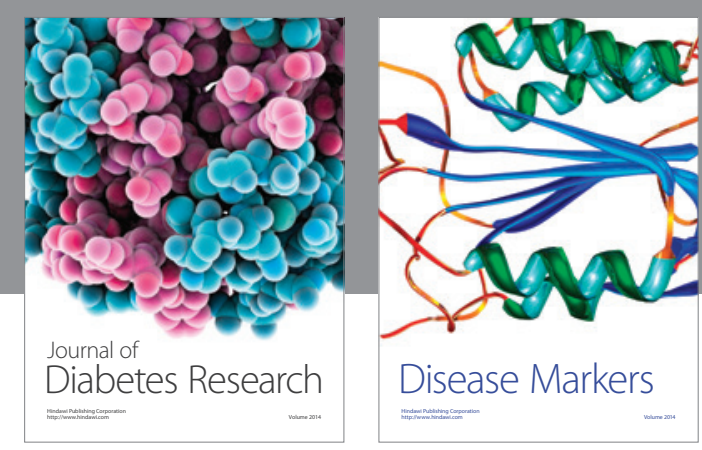

Disease Markers
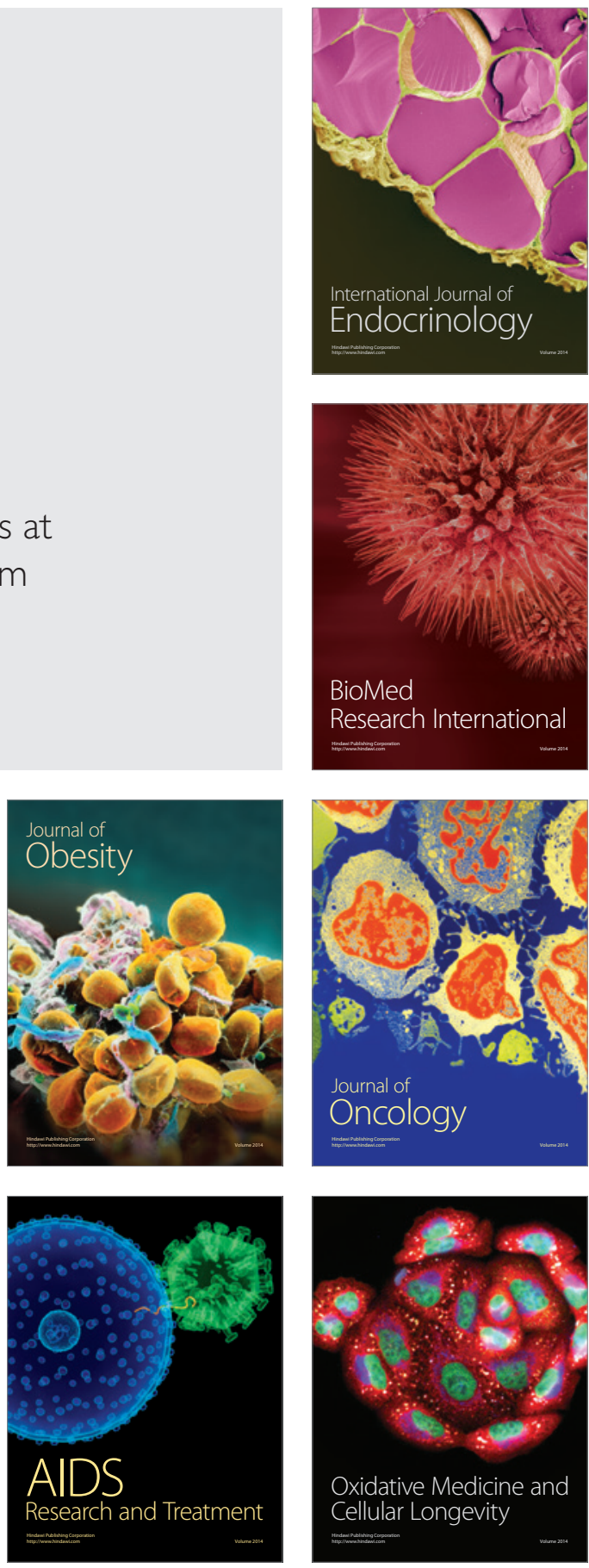